\title{
Evaluation of the forearm EMG signal features for the control of a prosthetic hand
}

\author{
Reza Boostani and Mohammad Hassan Moradi \\ Faculty of Biomedical Engineering, Amir Kabir University of Technology, Tehran, Iran
}

Received 1 July 2002, in final form 27 November 2003

Published 20 March 2003

Online at stacks.iop.org/PM/24/309

\begin{abstract}
The purpose of this research is to select the best features to have a high rate of motion classification for controlling an artificial hand. Here, 19 EMG signal features have been taken into account. Some of the features suggested in this study include combining wavelet transform with other signal processing techniques. An assessment is performed with respect to three points of view: (i) classification of motions, (ii) noise tolerance and (iii) calculation complexity. The energy of wavelet coefficients of EMG signals in nine scales, and the cepstrum coefficients were found to produce the best features in these views.
\end{abstract}

Keywords: wavelet transform, wavelet packet, cepstrum, cumulant, artificial neural network (ANN), integral absolute value (IAV), auto regressive (AR), principal component analysis (PCA)

\section{Introduction}

It has been proposed that the EMG signals from the body's intact musculature can be used to identify motion commands for the control of a prosthetic hand (Tucker and Liu 1999, Christodoulou and Pattichis 1999). Researchers have been working on this issue for several decades. The critical problem of these investigations is the choice and computation of effective features from the signals. They must permit the amputee's volitional muscle control to be monitored in a way that permits accurate estimation of the state of muscle activation. Further, the states of muscle activation must be mapped on to the desired prosthetic control operations. In this way, Graupe and Cine showed that a fourth-order time-series model of the EMG signals can be classified by a linear discrimination function (Graupe and Cine 1975), but this method involves a high complexity in computation. The results of Kelly and Parker's work illustrated that a Hopfield neural network could produce AR coefficients from the EMG signals in a shorter time (Kelly and Parker 1990). Furthermore, Saridis and Gootee presented integral absolute value and zero-crossing features that could produce appropriate feature space in order to classify arm motions (Saridis and Gootee 1983). Zardoshti et al (1995) extracted 
some features such as the integral of absolute value, variance, number of zero crossings and auto-regressive model parameters from upper limb EMG signals and then evaluated them with K-nearest neighbour (a non-parametric classifier). They presented a new feature, the EMG histogram, which is very suitable for the classification of hand motions, and also showed that this feature is appropriate to calculate both speed and noise tolerance. Chang et al (1994) used the variance of the rectified wave envelope and IAV features and Mahalonobius distance to classify four preshaping grasp movements. They also showed that these features could classify movements up to $90 \%$ accuracy. Kang et al (1995) compared AR and cepstrum coefficients and showed that the cepstrum coefficients are quite useful to improve classification rate. The time-frequency transform has also been introduced as a new mathematical approach to the time-frequency domain. Biomedical signals, especially EMG signals, have been processed by time-frequency transforms in order to extract more representative features to improve the rate of classification of motions. In this way, Jung et al (1994) imposed the WignerVille transform on the upper limb EMG signals to classify six different movements. Wellig and Moschytz (1999) also used packet wavelet transform to decompose EMG signals and reduced the misclassification rate. Liyu et al (1999) distinguished four forearm motions by decomposing two channels of EMG signals with a wavelet transform in six levels, and finally classified these coefficients by an ANN classifier. Abel et al (1998) by applying the inter-scale local maximum method on the wavelet coefficients of EMG signals presented new features, which improved the classification rate among neuropathic, myopathic and normal groups. Englehart et al (1999) extracted the upper limb EMG signals from four channels and then, by extracting wavelet coefficients, reduced their dimensions by the PCA transform, and finally the misclassification rate was decreased. Although the literature includes many papers which explore the extraction of features from the EMG for controlling prosthetic limbs, there have been few works which make quantitative comparison of their quality. Overall, a high quality EMG feature space should have the following properties:

Maximum class separability. A high quality feature space is that which results in clusters that have maximum class separability or minimum overlap. This ensures that the resulting misclassification rate will be as low as possible.

Robustness. The selected feature space should preserve the cluster separability in a noisy environment as much as possible.

Complexity. The computational complexity of the features should be kept low so that the related procedure can be implemented with reasonable hardware and in a real-time manner.

This paper is aimed at selecting the best features from the three viewpoints mentioned above. The result of this evaluation can be widely used in some applications such as EMG control of robots, prostheses and neuroprostheses. For this purpose, we evaluate features that have been used in the EMG signal processing methods and some new combined features in the wavelet domain. We first extract 19 features from EMG signals and evaluate them with evaluation criteria (i.e., Davies-Bouldin and scattering). Next, we propose the data acquisition method and, finally, we will show the results.

\section{Feature parameters}

Though a few features are defined in the time domain, most of them are defined in the frequency and time-frequency domains. The features used in this research are listed below: 
Integral of absolute value (IAV). The IAV of EMG is calculated as

$$
\mathrm{IAV}=\frac{1}{N} \sum_{i=1}^{N} x_{i}
$$

where $x_{i}$ is the $i$ th sample and $N$ is the number of samples in each segment.

Variance (VAR). The variance is a measure of the signal power and is calculated as

$$
\text { VAR }=\frac{1}{N-1} \sum_{i=1}^{N} x_{i}^{2}
$$

where $x_{i}$ is the $i$ th sample and $N$ is the number of samples in each segment.

Wilson amplitude (WAMP). This is the number of times that the difference between two consecutive amplitudes in a time segment becomes more than threshold (Hui and Park 1998). The threshold value is $50 \mu \mathrm{V}$. It can be formulated as

$$
\mathrm{WAMP}=\sum_{i=1}^{N} f\left(\left|x_{i}-x_{i+1}\right|\right)
$$

where

$$
f(x)= \begin{cases}1 & \text { if } x>\text { threshold } \\ 0 & \text { otherwise }\end{cases}
$$

This feature is an indicator of firing motor unit action potentials (MUAP) and therefore an indicator of the muscle contraction level.

Zero crossing $(\mathrm{ZC})$. Zero crossing is the number of times that the signal passes the zero amplitude axes (Chang et al 1996). It is calculated as

$$
\begin{aligned}
& \mathrm{ZC}=\sum_{i=1}^{N} \operatorname{sgn}\left(-x_{i} x_{i+1}\right) \\
& \operatorname{sgn}(x)= \begin{cases}1 & \text { if } x>0 \\
0 & \text { otherwise. }\end{cases}
\end{aligned}
$$

The number of turns (NT). This feature determines the number of changes in the sign of the slope in a time segment; in other words, the number of signal peaks in a time segment (Zardoshti et al 1995).

Mean of amplitude (MA). This feature determines the mean value of the difference in amplitudes of two consecutive samples in a time segment (Zardoshti et al 1995).

Wavelength. This feature estimates the length of the waveform in a segment and is defined as

$$
W(n)=\sum_{i=n-N+1}^{n}\left|\Delta x_{i}\right|
$$

where $\Delta x_{i}$ is defined as $\Delta x_{i}=x_{i}-x_{i-1}$.

Mean frequency. This feature estimates the mean frequency of the signal in a time segment (Park and Meek 1993).

Histogram. This feature determines the number of signal samples in different amplitude levels in a time segment, and was introduced by Zardoshti et al (1995). This feature is an extension 
of the ZC and WAMP measures, both of which compare a single threshold to the EMG signal or a closely related function. In this work the number of levels was set to 9 because the performance improved significantly as the number of levels was increased to 9 .

Auto-regressive coefficients $(A R)$. The simplest time-series model is the AR model, in which signal samples are estimated by linear combination of their earlier samples. It has been shown that the EMG spectrum changes with muscle contraction state, resulting in changes in AR coefficients (Graupe et al 1985). To define the order of the model, various experimental and theoretical approaches have been proposed (Philipson and Larsson 1988). In the experimental approach, the order $P$ is considered adequate when an increase in $P$ causes an empirically insignificant reduction in residual noise. Graupe and Cine showed that the model order $P=4$ is suitable for EMG signals (Graupe and Cine 1975), and we therefore used it in our research.

Auto-regressive coefficients were extracted from third-order cumulant. AR coefficients of order 4 are extracted from the third-order cumulant of the signal in a time segment. In this method, auto-correlation functions are replaced with the third-order cumulant in the Levinson-Durbin algorithm (Nikias 1993) that is computed as follows:

$$
c_{k x}(m, n)=\sum_{j=1}^{p} a_{j} c_{k x}(m-j, n)
$$

where $P$ is the model order, $m$ and $n$ are lag elements in two dimensions and $K$ is the interval index.

Auto-regressive coefficients were extracted from fourth-order cumulant. We extract AR coefficients of the fourth order from the fourth-order cumulant. The reason for calculating the AR coefficients by the third- and fourth-order cumulants is to obtain them in more representative features, which can determine the relations among samples in higher orders and also contain phase information on the signal.

Cepstral coefficients (Ceps). Cepstrum coefficients are a very powerful tool for speech applications (Cohen 1986). This feature can be calculated as

$$
c_{1}=a_{1} \quad c_{n}=-\sum_{k=1}^{n}\left(1-\frac{k}{n}\right) a_{k} c_{n-k}-a_{n}
$$

where $c_{i}$ is the $i$ th cepstrum coefficient and $a_{i}$ is the $i$ th auto-regressive coefficient. The most important characteristic of this feature is the deconvolution of a signal into two main parts so that each can be extracted by imposing a lifter in the cepstrum domain. It has been shown that this feature is very suitable for motion classification (Kang et al 1995).

Energy of wavelet coefficients of the EMG signal in nine scales. EMG signals were decomposed by wavelet transform into up to nine scales and the signal energy was then determined in nine scales as components of the feature vector. In this way, we used a bi-orthogonal mother wavelet, because this basis function had good matching with EMG signals. The reason for choosing nine scales is that the number of decomposition levels has a direct relation (dyadic) with the number of samples in each segment. In real-time functions, such as the control of a prosthetic hand, we are restricted to selecting a limited time for processing. In this paper the 
length of time segments has been selected as $200 \mathrm{~ms}$ and the number of samples in this time interval is 512. Therefore, we decomposed segments into the nine scales.

Energy of wavelet packet coefficients of the EMG signal in nine scales. EMG signals were decomposed by the wavelet packet transform into up to nine scales and signal energy was determined in nine scales as components of the feature vector. The advantage of the packet wavelet in comparison with the wavelet is that the wavelet packet transform decomposes the signal into both high and low frequencies in dyadic forms. Therefore, the number of feature components is increased, but the computation complexity is also increased.

Zero crossing of the wavelet coefficients of the EMG signal in nine scales. This feature is the number of base-line crossings in the wavelet domain in nine scales (Mallat 1991). Therefore, we have nine dimension vectors as the feature vectors. This feature is suggested in our approach.

IAV and ZC of wavelet coefficients of the EMG signal in nine scales. Mallat (1991) showed that the zero-crossing feature in the wavelet domain is a very unstable feature. Therefore to compensate for this defect, he suggested that if the area between each two crossings of the base level in the wavelet domain is added, we will have a stable feature. So we used IAV and zero-crossing features in nine scales in the wavelet domain as a feature vector which has 18 components.

Auto-regressive coefficients calculated by the third-order cumulant of wavelet coefficients. This feature is used in the time domain and its cluster situations are examined in the wavelet domain.

Auto-regressive coefficients which are calculated by the fourth-order cumulant of wavelet coefficients.

\section{Evaluation criteria}

Generally, the methods of feature evaluation are divided into two: (i) imposing features onto the classifier and measuring the misclassification rate, and (ii) evaluation of the feature space by statistical criteria. The first method has the disadvantage that the evaluation of the features depends on the classifier type (neural network, statistical classifier, neuro fuzzy classifier, etc). The second method is not problematic in this sense and tries to quantify the suitability of the feature space. There are many methods for feature evaluation in feature space such as Mahalonobius distance, Battacharia distance and maximum entropy (Fukunaga 1993). We used two parametric methods, which can evaluate features straightforwardly, and directly address the issue of class separability in the feature space. The most important advantage of these methods is that there is no need to suppose that the clusters have a normal distribution in the feature space.

\subsection{Davies-Bouldin (DB) criterion}

This criterion can estimate feature space quality. This parametric method is based on the scatter matrices of data (Davies and Bouldin 1979). It requires the computation of the clusterto-cluster distance. Then the worst condition for each cluster is determined and finally the DB index is obtained through averaging the worst case separation of each cluster from the others, 
Table 1. List of electrode positions and their specific muscles.

\begin{tabular}{ll}
\hline Channel 1 & Flexor Carpi Ulnaris \\
Channel 2 & Flexor Digitorum \\
Channel 3 & Flexor Carpi Radialis \\
Channel 4 & Brachio Radialis \\
Channel 5 & Extensor Carpi Radialis Longus \\
Channel 6 & Extensor Carpi Radialis Brevis \\
Channel 7 & Extensor Digitorum \\
Channel 8 & Extensor Carpi Ulnaris
\end{tabular}

Table 2. List of movements.

\begin{tabular}{ll}
\hline 1. Strong palmar flexion of wrist & 2. Weak palmar flexion of wrist \\
3. Strong extension of wrist & 4. Weak extension of wrist \\
5. Strong grasping & 6. Weak grasping \\
7. Strong abduction & 8. Weak abduction \\
9. Strong adduction & 10. Weak adduction \\
11. Pinch & 12. No motion \\
13. Thumb extension & 14. Supination \\
15. Pronation &
\end{tabular}

which is calculated as follows:

$$
R_{i, j}=\frac{S_{i}+S_{j}}{D_{i, j}}
$$

where $R_{i, j}$ is the value of the cluster-to-cluster distance and $K$ is the number of clusters (Davies and Bouldin 1979). $S_{i}$ and $S_{j}$ are the dispersions of the $i$ th and $j$ th clusters, respectively, and $D_{i, j}$ is the distance between their mean values. The DB index is defined as

$$
\mathrm{DB}=\frac{1}{K} \sum_{i=1}^{K} \max \left(R_{i, j}\right) \quad i \neq j .
$$

In essence, the DB index shows how badly the clusters may have overlap with their nearest neighbours. Lower values of the DB index imply a higher degree of cluster separability. The DB index is related to the performance of the linear Fisher discriminant classifier to pairwise clusters.

\subsection{Scattering criterion}

This criterion estimates the feature space quality but it differs essentially from the previous criterion, which compared the cluster situations two by two. This means the previous criterion cannot assess all clusters simultaneously, and can only give an averaging of the worst situation distances of two by two clusters. On the other hand, the scatter criterion considers clusters altogether, but it cannot report the exact situation of a cluster in comparison with the others. In order to calculate a formula that offers this criterion, first, the covariance matrix of betweencluster means is determined and then the covariance matrix of the classes is determined. To achieve the value of the scattering criterion, the trace of the covariance matrix of betweencluster means is divided into the trace of the summation of the covariance matrix of all classes (Fukunaga 1993). We define the matrix as follows:

$$
S_{w}=\sum_{i=1}^{k} p_{i} E\left(\left(x-m_{i}\right)\left(x-m_{i}\right)^{T}\right) .
$$


Table 3. The results of the feature evaluation from the three view points.

\begin{tabular}{|c|c|c|c|c|}
\hline Features & $\begin{array}{l}\text { Results of } \\
\text { evaluation by } \\
\text { scattering } \\
\text { criterion }\end{array}$ & $\begin{array}{l}\text { Results of } \\
\text { evaluation by } \\
\text { Davies-Bouldin } \\
\text { criterion }\end{array}$ & $\begin{array}{l}\text { Sensitivity of } \\
\text { feature space } \\
\text { to noise }\end{array}$ & $\begin{array}{l}\text { Calculation } \\
\text { time in } \\
\text { seconds }\end{array}$ \\
\hline 1. IAV & $1.3505 \times 10^{-4}$ & 40.9646 & 1.1392 & 0.00001 \\
\hline 2. Variance & 0.0016 & 66.9789 & 0.7976 & 0.0047 \\
\hline 3. Wilson points & $1.0223 \times 10^{-4}$ & 30.6471 & 0.3168 & 0.0180 \\
\hline 4. Zero crossing & $3.1223 \times 10^{-4}$ & 457.8970 & 0.7319 & 0.0133 \\
\hline 5. NT & $1.6992 \times 10^{-4}$ & 69.9430 & 0.5749 & 0.0707 \\
\hline 6. MA & $1.6973 \times 10^{-4}$ & 69.6420 & 0.6240 & 0.0733 \\
\hline 7. Wavelength & $1.1195 \times 10^{-4}$ & 51.5342 & 0.7781 & 0.0260 \\
\hline 8. Median frequency & 0.0268 & 35.6197 & 0.8955 & 0.0593 \\
\hline 9. Histogram & 0.0013 & 38.8624 & 0.3954 & 0.5860 \\
\hline 10. AR coefficients & $4.8073 \times 10^{-4}$ & 962.0877 & 1.5487 & 0.5740 \\
\hline $\begin{array}{l}\text { 11. AR coefficients from } \\
\text { third-order cumulant }\end{array}$ & 0.0451 & 16.1158 & 0.8341 & 0.1493 \\
\hline $\begin{array}{l}\text { 12. AR coefficients from } \\
\text { fourth-order cumulant }\end{array}$ & 0.0578 & 11.9674 & 3.8201 & 0.2427 \\
\hline 13. Cepstrum coefficients & 0.6774 & 1.9789 & 0.7231 & 0.0067 \\
\hline $\begin{array}{l}\text { 14. The energy of wavelet } \\
\text { coefficients of EMG signal } \\
\text { in nine scales }\end{array}$ & 2.7831 & 1.7843 & 0.1942 & 0.1100 \\
\hline $\begin{array}{l}\text { 15. The energy of wavelet } \\
\text { packet coefficients of EMG } \\
\text { signal in nine scales }\end{array}$ & 67.3726 & 0.1247 & 0.1134 & 10.2431 \\
\hline $\begin{array}{l}\text { 16. Zero crossing of wavelet } \\
\text { transform of signals } \\
\text { in nine scales }\end{array}$ & 0.3229 & 5.0129 & 122.1186 & 0.1747 \\
\hline $\begin{array}{l}\text { 17. IAV and ZC of wavelet } \\
\text { coefficients of EMG signal } \\
\text { in nine scales }\end{array}$ & $1.3505 \times 10^{-4}$ & 10.3295 & 3.0186 & 0.0001 \\
\hline $\begin{array}{l}\text { 18. Auto-regressive coefficients, } \\
\text { extracted from third-order } \\
\text { cumulant of wavelet coefficients }\end{array}$ & 0.0071 & 21.3787 & 15.1732 & 0.7580 \\
\hline $\begin{array}{l}\text { 19. Auto-regressive coefficients, } \\
\text { extracted from fourth-order } \\
\text { cumulant of wavelet coefficients }\end{array}$ & 0.0169 & 12.3694 & 25.3436 & 1.1680 \\
\hline
\end{tabular}

$S_{w}$ is the covariance matrix of all classes in which $m_{i}$ is the mean of the $i$ th class and $x$ is the sample vector.

$$
S_{B}=\sum_{i=1}^{k} p_{i}\left(m_{i}-m_{\text {mean }}\right)\left(m_{i}-m_{\text {mean }}\right)^{T} .
$$

$S_{B}$ is the covariance matrix of between-classes means in which $m_{\text {mean }}$ is the mean of all the classes means. Finally, the value of the scattering criterion is calculated as

$$
J=\operatorname{trace}\left(S_{w}\right) / \operatorname{trace}\left(S_{B}\right) .
$$

It is obvious that the quality of the space feature will improve when the value of the criterion decreases. 


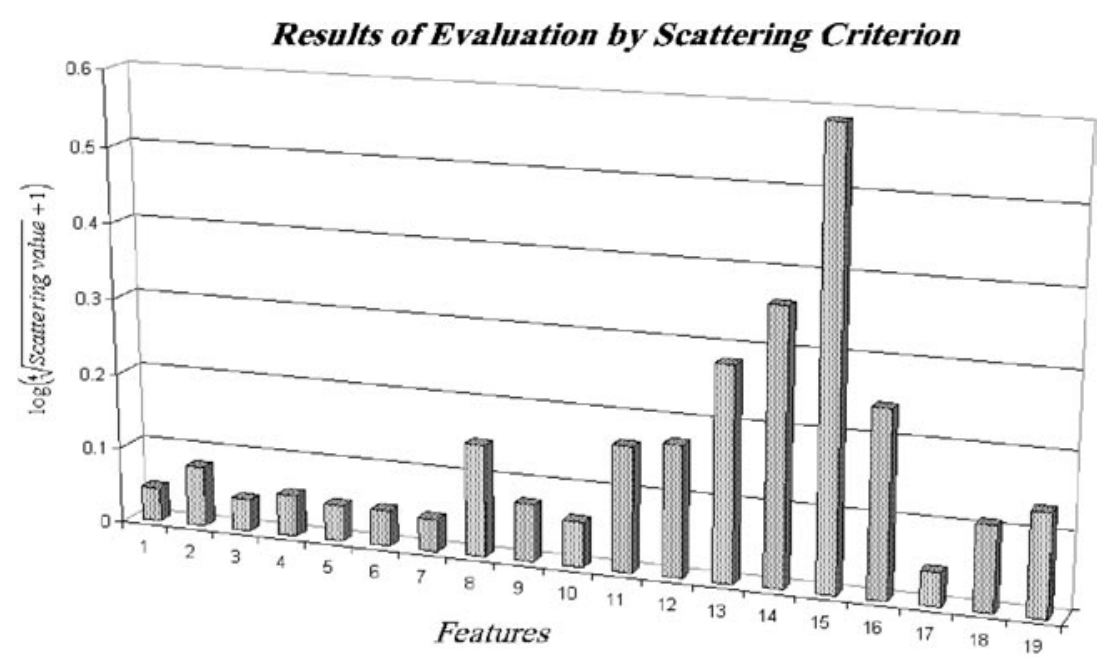

Figure 1. The evaluation of 19 features by scattering criterion. To show the differences on a better scale, the non-linear transform (log) is imposed on the scattering values.

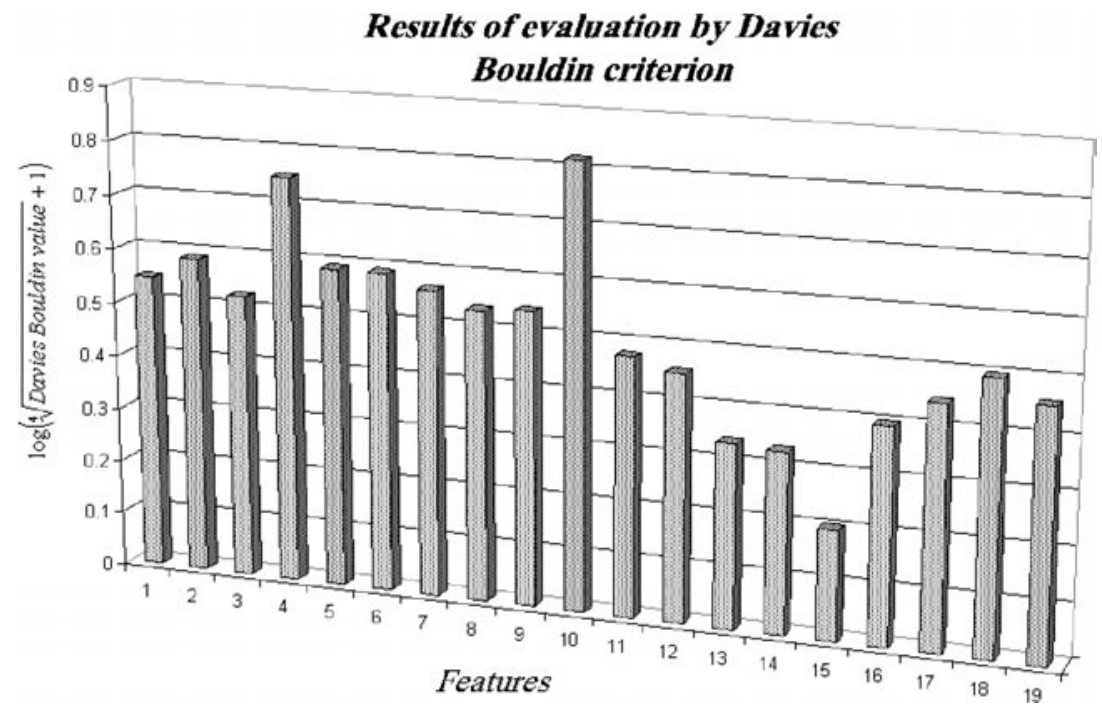

Figure 2. The evaluation of 19 features by the Davies-Bouldin criterion. To show the differences on a better scale, the non-linear transform $(\log )$ is imposed on the scattering values.

\section{Data acquisition and data processing}

The signals were taken from ten disabled people. Each person had a hand amputated at the wrist and their ages ranged from 25 to 35 . They were asked to sit at the platform and try to imagine some movements, which are listed in table 2. During this procedure, EMG signals were taken from eight gold electrodes that contacted anatomically on eight effective muscles of their forearms (which are involved with the related movements). The set-up in the laboratory of the Medical Physics faculty of Tarbiat Modares University was used to take the signals. The muscles and related channels are listed in table 1. 
Sensitivity of Features Against Noise

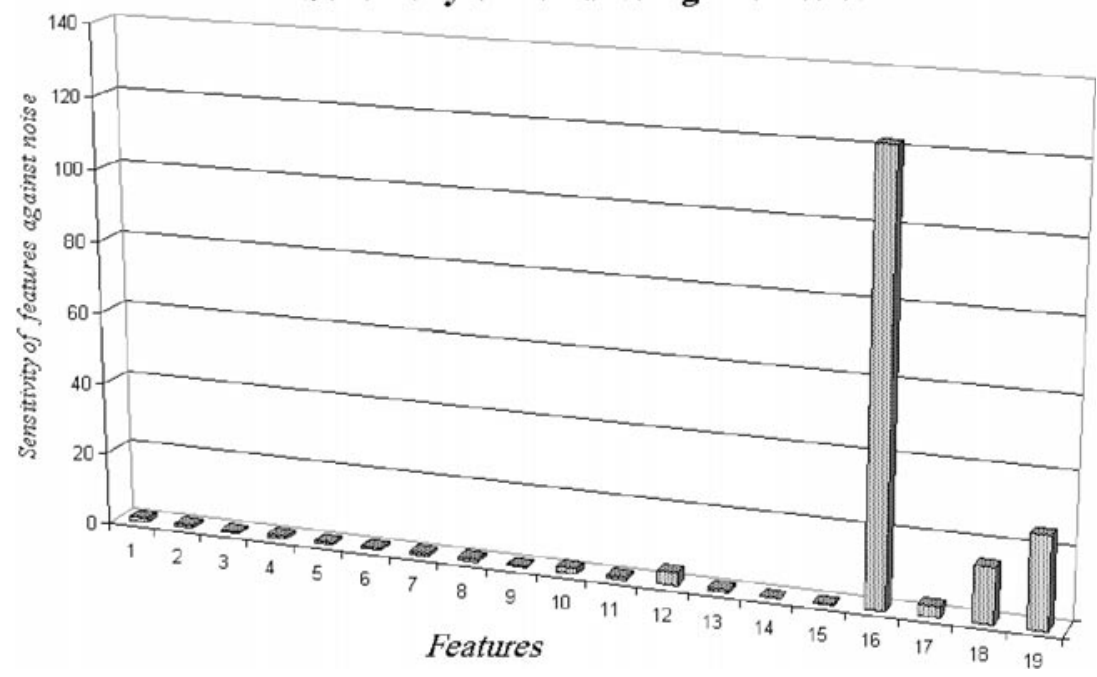

Figure 3. The sensitivity of 19 features against simulated noise.

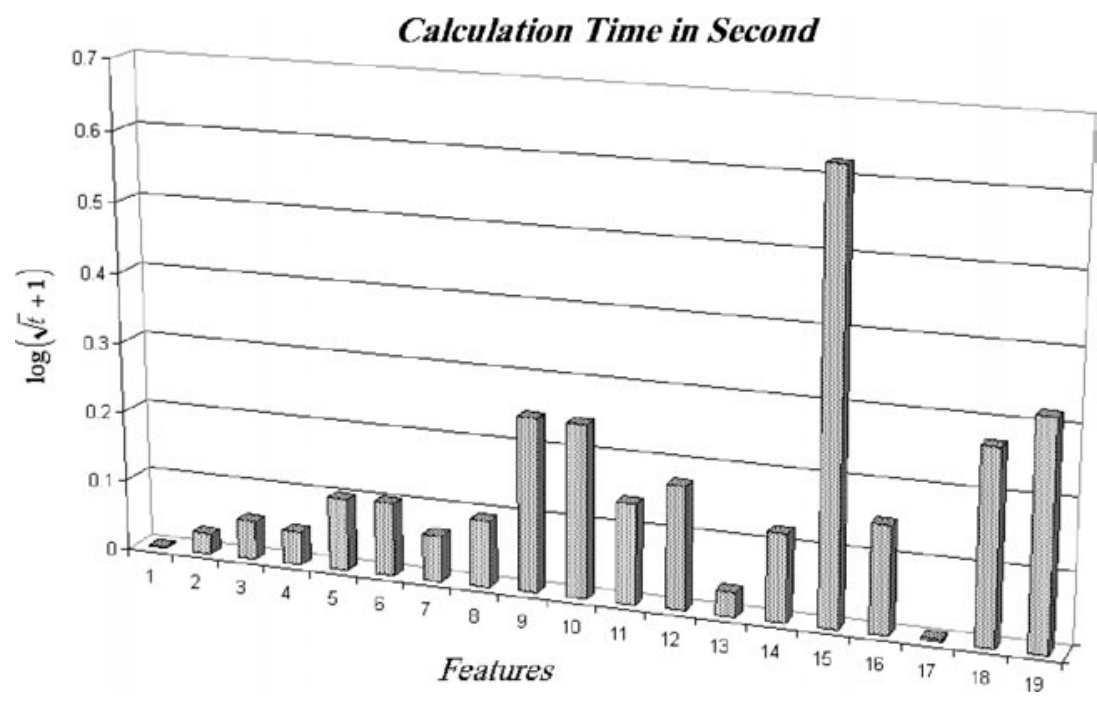

Figure 4. The evaluation of features from the complexity of computation view point.

To avoid cross talk between channels, the diameter of the electrodes selected was $6 \mathrm{~mm}$. The instrument had eight channels and the sampling rate was selected to be $2975 \mathrm{~Hz}$. Both the first and the final parts of the signals were truncated to obtain reliable signals. The latter improvement was achieved through avoidance of fatigue effects. Signals were passed through the nine-order Butterworth low pass filter to enhance their quality. The patients were asked to repeat each motion 20 times in order to get significant results. This resulted in a statistically significant set from the samples. The $F$-test was performed to assure that there was significant difference between the results of the evaluation features. The $T$-test was then imposed between 
every two sets of feature evaluations. Finally, the patients were asked to perform 15 defined movements, which are listed in table 2.

To have significant results from feature evaluation, the $F$-test and $T$-test were performed on scattering and Davies-Bouldin results. All results were significant, which means in other words that the $p$ value has been lower than 0.05 for all evaluation results. For processing the data, we first considered 512 samples or $200 \mathrm{~ms}$ for window length. Window overlap was considered to be $50 \%$, and features were extracted from segments, and finally the above criteria were applied to the features.

\section{Results}

The results of the feature evaluation from the three view points of movement classification, noise tolerance and calculation complexity are given in table 3. Four graphs are depicted to clarify results from table 3 in figures 1, 2, 3 and 4 respectively on different scales. Noise sensitivity of a feature space is described through noise tolerance. Noise usually involves disturbance movements with low frequency and also line noise. If the value of this sensitivity is very low, it could be judged that the feature is very robust against the noise. To simulate real noise, two different noises are considered, where one of them simulates the electrical power supply $(50 \mathrm{~Hz})$, and another one is a random noise. The amplitude of each noise is one tenth of the amplitude of the peak-to-peak range of the EMG signals. Calculation complexity is an important factor in online applications, particularly in artificial hand control. The results illustrate that the features of energy of wavelet packet coefficients, energy of wavelet coefficients and cepstrum coefficients of EMG signals present the best results from the classification and noise tolerance view points respectively (table 3). Although wavelet packet transform leads to the best results for these two approaches, it drops dramatically through calculating run time. Over all, the energy of wavelet coefficients of EMG signal in nine scales shows the best result through averaging compared to the others.

\section{Conclusion}

High rates of classification of movements with respect to the classification rate, noise tolerance and computational complexity were evaluated from the optimum and effective features view points. Nineteen features are used to evaluate EMG signals in order to control a prosthetic hand. The results presented here have also important implications to other applications controlled by EMG such as robotics or neuroprosthetic devices. Two statistical criteria including DaviesBouldin and scattering criteria were used to increase estimation of feature space quality. The Davies-Bouldin criterion considers cluster situations two by two, and the scattering criterion covers all cluster situations together. Both criteria yielded similar results.

The $F$-test and $T$-test were applied to features to assure result validation. The $p$ value was less than 0.05 in all results.

The results indicate that the energy of wavelet coefficients in nine scales and cepstrum coefficients, which were selected in the evaluation procedure, lead to the best features.

\section{Acknowledgments}

The authors wish to warmly express their appreciation to Dr Firoozabadi, Mr Makkiabadi, Mr Ghoddami and Mrs Lotfi for their assistance in obtaining signals from disabled persons. 


\section{References}

Abel R, Arikidis N A and Forster A 1998 Inter-scale local maxima-a new technique for wavelet analysis for EMG signals Proc. 20th Ann. Int. Conf. IEEE Engineering in Medicine and Biology Society (Hong Kong, 1998)

Chang G, Kang W, Luh J and Cheng C 1994 Principal component for classification of preshaping movement IEEE, 17th Ann. Conf. pp 1019-25

Chang G C, Jan W, Junn J and Kung C 1996 Real time implementation of electromyogram pattern recognition as a control command of man-machine interface IEEE Conf. on Med. \& Eng. Physics vol 18 pp 527-37

Christodoulou C I and Pattichis C S 1999 Unsupervised pattern recognition for the classification of EMG signals IEEE Trans. Biomed. Eng. 46 169-78

Cohen A 1986 Biomedical Signal Processing (Boca Raton, FL: CRC Press)

Davies D L and Bouldin D W 1979 A cluster separation measure IEEE Pattern Anal. Mach. Intell. 1 224-7

Englehart K, Hudgins J L, Parker P and Stevenson G 1999 Improving myoelectric signal classification using wavelet packets and principal components analysis IEEE, Proc. 1st Joint BMES/EMBS Conf. Serving Humanity p 569

Fukunaga K 1993 Statistical Pattern Recognition (Englewood Cliffs, NJ: Prentice-Hall)

Graupe J and Cine K 1975 Functional separation of EMG signals via ARMA identification method for prosthesis control purpose IEEE Trans. Syst. Man Cybern. 5 252-9

Graupe J, Salahi J and Zhang D 1985 Stochastic analysis of electromyogram temporal signatures for multi functional signal-site activation of prostheses and orthoses J. Biomed. Eng. 7 18-28

Hui S and Park I 1998 EMG pattern recognition based on artificial intelligent techniques IEEE Trans. Rehabil. Eng. $6400-5$

Jung G C, Shin J and Sankuo T 1994 Using time frequency analysis technique in the classification of surface EMG signals Proc. IEEE in Medicine and Biology

Kang W, Shiu J, Cheng C K and Shin J 1995 Real time implementation of cepstrum coefficient and maximum likelihood method in EMG pattern recognition IEEE Trans. Biomed. Eng. 42 777-85

Kelly M and Parker P 1990 Application of neural network to myoelectric signal analysis, preliminary study IEEE Trans. Biomed. Eng. 37 221-30

Liyu C, Zhizhong W and Haihong Z 1999 An EMG classification methods on wavelet transform IEEE, Proc. 1st Joint BMES/EMBS Conf. Serving Humanity p 565

Mallat S 1991 Zero crossing of a wavelet transform IEEE Trans. Inf. Theory 37 1019-33

Nikias L 1993 Higher Order Spectra Analysis: a Nonlinear Signal Processing Framework (Englewood Cliffs, NJ: Printice-Hall)

Park E and Meek S 1993 Fatigue compensation of the electromyogram signal for prosthetic control and forced estimation IEEE Trans. Biomed. Eng. 40 1019-23

Philipson L and Larsson P G 1988 The electromyogram signal as measure for muscular force: a comparison of detection and quantification techniques J. Electromyogram. Clin. Neurophysiol. 28 141-50

Saridis G and Gootee T 1983 EMG pattern analysis and classification for a prosthetic ARMA IEEE Trans. Biomed. Eng. 30 18-29

Tucker C A and Liu X 1999 Self-organizing maps for time series analysis of electromyographic data IEEE Neural Netw. 5 3577-80

Wellig P and Moschytz G S 1999 Classification of time-frequency atoms IEEE Conf. p 953

Zardoshti M, Wheeler B, Badie K and Hashemi M R 1995 EMG feature evaluation for movement control of upper extremity prostheses IEEE Trans. Rehabil. Eng. 3 324-33 